\title{
CONJUGACY IN NORMAL SUBGROUPS OF HYPERBOLIC GROUPS
}

\author{
ARMANDO MARTINO AND ASHOT MINASYAN
}

\begin{abstract}
Let $N$ be a finitely generated normal subgroup of a Gromov hyperbolic group $G$. We establish criteria for $N$ to have solvable conjugacy problem and be conjugacy separable in terms of the corresponding properties of $G / N$. We show that the hyperbolic group from F. Haglund's and D. Wise's version of Rips's construction is hereditarily conjugacy separable. We then use this construction to produce first examples of finitely generated and finitely presented conjugacy separable groups that contain non-(conjugacy separable) subgroups of finite index.
\end{abstract}

\section{INTRODUCTION}

One of the most intuitive ways to study an infinite (discrete) group, $G$, is to look at its finite quotients. However, in general, one loses information about $G$ if one restricts attention to these finite quotients, and so arises the definition of residual finiteness, where $G$ is said to be residually finite if for every $x \neq y \in G$ there is homomorphism $\psi: G \rightarrow Q$, where $Q$ is a finite group, such that $\psi(x) \neq \psi(y)$ in $Q$.

To use this method more systematically, one introduces the profinite topology $\mathcal{P} \mathcal{T}(G)$ on $G$. This is the topology whose basic open sets are cosets of finite index normal subgroups in $G$. It is not difficult to check that the group operations are continuous with respect to this topology, thus $G$, equipped with $\mathcal{P} \mathcal{T}(G)$, is a topological group. Residual finiteness for $G$ then becomes the statement that this topology is Hausdorff; in fact, it is equivalent to the statement that singletons are closed.

A subset $A \subseteq G$ is said to be separable in $G$ if $A$ is closed in $\mathcal{P} \mathcal{T}(G)$. Intuitively, this means that $A$ can be recognized by looking at finite quotients of $G$. A group is called subgroup separable (or LERF) if every finitely generated subgroup is closed in $\mathcal{P} \mathcal{T}(G)$. Similarly, a group is called conjugacy separable if for every element $g \in G$, its conjugacy class $g^{G}:=\left\{h^{-1} g h \mid h \in G\right\} \subseteq G$ is closed in $\mathcal{P} \mathcal{T}(G)$. Equivalently, $G$ is conjugacy separable if and only if for any two non-conjugate elements $x, y \in G$ there exists a homomorphism $\psi$ from $G$ to a finite group $Q$ such that $\psi(x)$ is not conjugate to $\psi(y)$ in $Q$.

One can formalize the sense in which subsets closed in $\mathcal{P} \mathcal{T}(G)$ are "recognizable", and this was done classically by A. Mal'cev [22], who proved that a finitely presented residually finite group has solvable word problem. Mal'cev [22] also proved that a finitely presented

2000 Mathematics Subject Classification. 20F67, 20F10, 20E26.

Key words and phrases. Hereditary conjugacy separability, normal subgroups of hyperbolic groups, Rips's construction. 
conjugacy separable group has solvable conjugacy problem. (In fact, one can show that any recursively enumerable subset of a finitely presented group $G$, which is closed in $\mathcal{P} \mathcal{T}(G)$, has decidable membership problem.) Thus residual finiteness and conjugacy separability can be viewed as natural "profinite analogues" of the solvability of word and conjugacy problems respectively.

This analogy between decision problems and closure properties can be seen as part of the motivation for this paper. Namely, it is well known that if a (finitely presented) group has solvable word problem, then so does any finite index subgroup and any finite extension. Similarly, for a residually finite group, any finite index subgroup and any finite extension is also residually finite. However, one cannot say the same about the conjugacy problem: Collins and Miller [9] proved that there exists a finitely presented group with solvable conjugacy problem, with a finite index subgroup having unsolvable conjugacy problem. In the same paper [9] (see also [15]), they also construct a finitely presented group with solvable conjugacy problem and its finite extension with unsolvable conjugacy problem. The purpose of this paper is to provide a profinite analogue of the first of these. Specifically, our main theorem is

Theorem 1.1. For every integer $m \geq 2$ there exists a finitely presented conjugacy separable group $T$ that contains a non-(conjugacy separable) subgroup $S$ of index $m$. Moreover, $T$ can be chosen in such a way that

- $T$ is a subgroup of some right angled Artin group;

- both $T$ and $S$ have solvable conjugacy problem.

So while we would like conjugacy separability to pass to finite index subgroups, these examples show that it does not. For this reason, in [8] S. Chagas and P. Zalesskii defined a group $G$ to be hereditarily conjugacy separable if every finite index subgroup of $G$ is conjugacy separable, and constructed the first (infinitely generated) example of a conjugacy separable but not hereditarily conjugacy separable group. The concept of hereditary conjugacy separability is essential in the current paper. We believe that this concept is stronger (according to the theorem above) and more useful than simply conjugacy separability, in view of many applications, discovered in [25].

Let us also mention that an example of a finitely generated (but not finitely presented) non-(conjugacy separable) group $G$, containing a conjugacy separable subgroup $H$ of index 2, was constructed much earlier by A. Goryaga in [14. As it can be easily seen from Goryaga's argument, the conjugacy problem is unsolvable in this group $G$.

In order to briefly describe the strategy of our proof and the structure of the paper, let us start with the following

Theorem 1.2. Let $H$ be a finitely generated torsion-free normal subgroup of a hyperbolic group $G$. Then $H$ has solvable conjugacy problem if and only if $G / H$ has solvable word problem.

The above statement was probably known to the experts before this work. In one direction, it can be compared, for instance, with the result of M. Bridson [6], claiming that 
a normal subgroup $N$ of a bicombable group $G$ has solvable conjugacy problem, provided the generalized word problem is solvable in $G / N$; in the other direction it follows from [7. III., , Lemma 5.18]. We give a proof of Theorem 1.2 in Section 3. We include it here mainly to motivate Theorem 1.3 below, in which the word and conjugacy problems are replaced by the corresponding properties of the profinite topology.

Theorem 1.3. Suppose that $G$ is a torsion-free hereditarily conjugacy separable hyperbolic group and $H \triangleleft G$ is a finitely generated normal subgroup. Then $H$ is conjugacy separable if and only if $G / H$ is residually finite.

As one can see, in Theorem 1.3 we had to impose an additional assumption demanding that $G$ be hereditarily conjugacy separable. Presently, it is not known whether there exist non-(conjugacy separable) or non-(residually finite) hyperbolic groups. But if, for instance, there were a hyperbolic group $G$ that is not residually finite, then the trivial subgroup $H:=\{1\}$ would be conjugacy separable but $G / H \cong G$ would not be residually finite. Thus the statement of Theorem 1.3 would be false without that additional assumption.

In Section 5 we suggest a generic method for constructing a hyperbolic group $G$ and its finitely generated normal subgroup $H$ satisfying the assumptions of Theorem 1.3 . This method is based on the modification of Rips's construction, suggested by F. Haglund and D. Wise in [18], and on the result that right angled Artin groups are hereditarily conjugacy separable, which was established in [25]. We apply this hereditarily conjugacy separable version of Rips's construction together with Theorem 1.3 in Section 6, to give first examples of finitely generated conjugacy separable groups which contain non-(conjugacy separable) finite index subgroups.

In Section 7 we obtain similar criteria for conjugacy separability of fibre products, associated to normal subgroups of hyperbolic groups. And in Section 8 we construct the examples demonstrating our main Theorem 1.1 .

Acknowledgements. We would like to thank M. Belolipetsky, M. Bridson, F. Haglund and D. Wise for enlightening discussions.

\section{Preliminaries}

Let $(\mathcal{X}, d)$ be a geodesic metric space and let $\delta$ be a non-negative real number. $\mathrm{A}$ geodesic triangle $\Delta$ in $\mathcal{X}$ is said to be $\delta$-slim, if each of its sides is contained in the closed $\delta$-neighborhood of the union of the two other sides. The space $\mathcal{X}$ is called Gromov hyperbolic if there exists $\delta \geq 0$ such that every geodesic triangle $\Delta$ in $\mathcal{X}$ is $\delta$-slim. A subset $Q \subseteq \mathcal{X}$ is said to be $\varepsilon$-quasiconvex (for some $\varepsilon \geq 0$ ), if any geodesic connecting two elements from $Q$ belongs to a closed $\varepsilon$-neighborhood of $Q$ in $\mathcal{X}$.

Given a group $G$, generated by a finite symmetrized (i.e., $\mathcal{A}=\mathcal{A}^{-1}$ ) set of elements $\mathcal{A} \subset$ $G$, the corresponding Cayley graph $\Gamma(G, \mathcal{A})$ can be equipped with the natural simplicial metric, making it a proper geodesic metric space. The group $G$ is (Gromov) hyperbolic if its Cayley graph $\Gamma(G, \mathcal{A})$ is a Gromov hyperbolic metric space. A subset $Q \subseteq G$ is called 
quasiconvex if there is $\varepsilon \geq 0$ such that $Q$ is $\varepsilon$-quasiconvex, when regarded as a subset of $\Gamma(G, \mathcal{A})$.

Hyperbolic groups became a major subject of study in Geometric Group Theory since they were introduced by M. Gromov in [16]. Hyperbolicity of a given group $G$ does not depend on the choice of a particular finite generating set $\mathcal{A}$ of $G$. In the case when $G$ is hyperbolic, quasiconvexity of some subset $Q \subseteq G$ is also independent of $\mathcal{A}$. For this and other basic properties of hyperbolic groups the reader is referred to [13] and [1].

It is well known that hyperbolic groups have solvable word and conjugacy problems (see, for example, [7, III.Г.2.8]).

Assume, now, that $G$ is a hyperbolic group, $H \leq G$ is a subgroup and $S \subseteq G$ is a subset. The centralizer $C_{H}(S)$, of $S$ in $H$, is the subgroup $\{h \in H \mid h s=s h, \forall s \in S\} \leq G$. It is a basic fact that for a hyperbolic group, $G$, and each infinite order element $g \in G$, the cyclic subgroup $\langle g\rangle$ has finite index in the centralizer $C_{G}(g)$ (see [13, 8.3.34]).

The subgroup $H \leq G$ is called elementary if it contains a cyclic subgroup of finite index. Every infinite order element $g \in G$ belongs to a unique maximal elementary subgroup $E_{G}(g) \leq G$ (see, for instance, [27, Lemma 1.16]). In particular, $C_{G}(g) \subseteq E_{G}(g)$.

As A. Olshanskii showed in [27, Prop. 1], for every non-elementary subgroup $H \leq G$ there is a unique maximal finite subgroup $E_{G}(H)$ normalized by $H$ in $G$. More precisely, $E_{G}(H)=\bigcap_{g \in H^{0}} E_{G}(g)$, where $H^{0}$ denotes the subset of all infinite order elements in $H$. It is not difficult to see that for a non-elementary subgroup $H \leq G$ one has $C_{G}(H) \leq E_{G}(H)$.

Let $\Gamma$ be a finite simplicial graph, and let $\mathcal{V}$ and $\mathcal{E}$ be the sets of vertices and edges of $\Gamma$ respectively. The right angled Artin group $G$, associated to $\Gamma$, is given by the presentation

$$
G:=\langle\mathcal{V} \| u v=v u \text {, whenever } u, v \in \mathcal{V} \text { and }(u, v) \in \mathcal{E}\rangle \text {. }
$$

Let $G$ be a group and $H \leq G$. Then $H$ is said to be a virtual retract of $G$ if there is a finite index subgroup $K \leq G$ such that $H \leq K$ and $H$ is a retract of $K$ (that is, there exists an endomorphism $\rho: K \rightarrow K$ satisfying $\rho(K)=H$ and $\rho \circ \rho=\rho$ ).

The following two classes of groups were introduced in [25]. The first class $\mathcal{V} \mathcal{R}$ consists of all groups that are virtual retracts of right angled Artin groups. A group $G$ belongs to the second class $\mathcal{A} \mathcal{V} \mathcal{R}$, by definition, if $G$ contains a finite index subgroup $H \leq G$ with $H \in \mathcal{V} \mathcal{R}$.

\section{Conjugacy problem For Subgroups}

Proposition 3.1. Let $G$ be a hyperbolic group and $H$ a finitely generated torsion-free subgroup of $G$. If $H$ has solvable membership problem in $G$, then it also has solvable conjugacy problem.

Proof. Consider arbitrary $x, y \in H$. As $G$ is hyperbolic, we can decide if $x, y$ are conjugate in $G$. If they are not, then neither are they conjugate in $H$. If they are conjugate in $G$ we may, by enumeration, find a conjugator $g \in G$ such that $x^{g}:=g^{-1} x g=y$ (note 
that this relies on $G$ being finitely generated and having solvable word problem). It is then clear that $x, y$ are conjugate in $H$ if and only if $g \in C_{G}(x) H$. However, since $H$ is torsion free, $C_{G}(x)$ must be virtually cyclic. Therefore, the subgroup generated by $x$ has finite index in $C_{G}(x)$, and, furthermore, it is possible to algorithmically find a finite set of coset representatives for $\langle x\rangle$ in $C_{G}(x)$ (see [3, Prop. 4.11]). We shall call these $h_{1}, \ldots, h_{k}$. Therefore, $x$ and $y$ are conjugate in $H$ if and only if $h_{i}^{-1} g \in H$ for some $i$. Since the hypothesis allows us to decide this, we have solved the conjugacy problem in $H$.

Remark 3.2. The assumption that $H$ is finitely generated in Proposition 3.1 is not really needed: the set of words representing elements from $H$ is recursive because there is an algorithm $\mathfrak{A}$ that solves the membership problem to $H$ in $G$.

Proposition 3.3. Let $G$ be a hyperbolic group and $H$ a finitely generated subgroup of $G$. If $H$ is normal and has solvable conjugacy problem, then it also has solvable membership problem in $G$.

Proof. If $H$ is finite, the claim follows from the solvability of the word problem in $G$. So we may assume that $H$ is infinite. In this case there exists an element $x \in H$ of infinite order (by [13, 8.3.36]). Consequently, $\langle x\rangle$ has finite index in $C_{G}(x)$ and so $C_{H}(x)$ has finite index in $C_{G}(x)$. Thus there exists a finite collection of elements $1=f_{1}, f_{2}, \ldots, f_{k}$ in $G$, which are the right coset representatives of $C_{H}(x)$ in $C_{G}(x)$. Since the element $x$ is fixed throughout, we may assume that these are given (as words in the generators of $G$ ).

Consider some $g \in G$; we need to decide whether or not it lies in $H$. Since $H$ is normal, $x^{g} \in H$. Clearly, if $x^{g}$ is not conjugate to $x$ in $H$, then $g \notin H$ and we are done. Otherwise, enumerating all elements of $H$, we will be able to find an $h \in H$ such that $x^{g}=x^{h}$. Hence $g h^{-1} \in C_{G}(x)$ and $g \in H$ if and only if $g h^{-1} \in C_{H}(x)$. Now we know that $g h^{-1} f_{i}^{-1} \in H$ for some $i$. As $H$ is finitely generated and $G$ has solvable word problem, we may enumerate all elements of $H$, and for each $i$ check whether $g h^{-1} f_{i}^{-1}$ is equal to this element of $H$. By construction, this process is guaranteed to terminate after finitely many steps, and $g \in H$ if and only if the process tells us that $g h^{-1} f_{1}^{-1} \in H$.

Proof of Theorem 1.2. It is easy to see that for a normal subgroup $H$ of a group $G$, the membership problem to $H$ in $G$ is equivalent to the word problem in the quotient $G / H$. Therefore, the claim of Theorem 1.2 immediately follows from Propositions 3.3 and 3.1.

\section{Conjugacy Separable Subgroups}

As Mal'cev proved in [22, if $H$ is a finitely generated separable subgroup of a finitely presented group $G$, then the membership problem to $H$ in $G$ is solvable. Therefore one can see that the next fact is a "profinite analogue" of Proposition 3.1.

Proposition 4.1. Let $H$ be a torsion-free subgroup of a hyperbolic group $G$. If $G$ is hereditarily conjugacy separable and $H$ is separable in $G$ then $H$ is conjugacy separable. 
Proof. Consider any elements $x, y \in H$ that are not conjugate in $H$. Without loss of generality, we may assume that $x \neq 1$. Then $x$ must have infinite order, by our hypothesis, and therefore $\left|C_{G}(x):\langle x\rangle\right|<\infty$. Let $L:=C_{H}(x)=C_{G}(x) \cap H$, then $x \in L$ and one can find elements $z_{1}, \ldots, z_{k} \in C_{G}(x) \backslash H$ such that $C_{G}(x)=L \sqcup \bigsqcup_{i=1}^{k} z_{i} L$.

Since $H$ is separable in $G$, there exists a finite index subgroup $K \leq G$ such that $H \leq K$ and $z_{i} \notin K$ for every $i=1, \ldots, k$. Observe that, by construction, the centralizer $C_{K}(x)$, of $x$ in $K$, is contained in $H$ (in fact, it is equal to $L$ ).

Assume, first, that $y=g x g^{-1}$ for some $g \in K$. Since $x, y$ are not conjugate in $H$ we know that $g \notin H$. Also, $H$ is closed in $\mathcal{P} \mathcal{T}(K)$ because $H$ is closed in $\mathcal{P} \mathcal{T}(G)$ and $K \leq G$. Hence, there is a finite index subgroup $M \leq K$ such that $H \leq M$ and $g \notin M$. We claim that $x$ is not conjugate to $y$ in $M$. Indeed, otherwise, there would exist $u \in M$ with $u x u^{-1}=y=g x g^{-1}$, implying that $u^{-1} g \in C_{K}(x) \leq H$, i.e., $g \in u H \subseteq M$, which contradicts the choice of $M$.

Thus we proved that there is a finite index subgroup $N \leq G$ such that $H \leq N$ and $x$ is not conjugate to $y$ in $N$. Using the conjugacy separability of $N$, we can find a finite group $Q$ and a homomorphism $\varphi: N \rightarrow Q$ such that $\varphi(x)$ is not conjugate to $\varphi(y)$ in $Q$. And since $H \leq N$, the restriction of $\varphi$ to $H$ gives a finite quotient of $H$, in which the images of $x$ and $y$ are not conjugate.

Next comes a natural analogue of Proposition 3.3. Observe that, unlike the previous statement, it does not need to assume that $G$ is hereditarily conjugacy separable, but requires the subgroup $H$ to be normal and finitely generated.

Proposition 4.2. Let $H$ be a finitely generated non-elementary normal subgroup of a hyperbolic group $G$, with $E_{G}(H)=\{1\}$. If $H$ is conjugacy separable then $G / H$ is residually finite.

The proof of Proposition 4.2 makes use of the three lemmas below. The next statement is well-known and can be verified in a straightforward manner (see, for example, [24, Lemma 5.2]).

Lemma 4.3. Assume $G$ is a group and $H \triangleleft G$ is a normal subgroup such that $C_{G}(H) \subseteq H$. Then the quotient-group $G / H$ embeds into the outer automorphism group Out $(H)$.

An automorphism $\psi$ of a group $G$ is said to be pointwise inner, if for each $g \in G$, $\psi(g)$ is conjugate to $g$ in $G$. It is not difficult to see that the set of all pointwise inner automorphisms forms a normal subgroup $A u t_{p . i .}(G) \triangleleft A u t(G)$, and $\operatorname{Inn}(G) \leq A u t_{\text {p.i. }}(G)$. The following criterion was found by E. Grossman in [17, Thm. 1]:

Lemma 4.4. Let $H$ be a finitely generated conjugacy separable group with Aut $_{\text {p.i. }}(H)=$ $\operatorname{Inn}(H)$. Then $\operatorname{Out}(H)$ is residually finite.

The last ingredient is given by [26, Cor. 5.4], where D. Osin and the second author showed that $\operatorname{Aut}_{\text {p.i. }}(H)=\operatorname{Inn}(H)$ for any non-elementary subgroup $H$ of a relatively hyperbolic group $G$, provided $H$ contains at least one infinite order element that is not 
conjugate to an element of a parabolic subgroup in $G$, and $E_{G}(H)=\{1\}$. Since every hyperbolic group is relatively hyperbolic with respect to the trivial subgroup (see [28]), and an infinite subgroup of a hyperbolic group necessarily contains an element of infinite order $([13,8.3 .36])$, we obtain

Lemma 4.5. If $H$ is a non-elementary subgroup of a hyperbolic group $G$ with $E_{G}(H)=$ $\{1\}$, then Aut p.i. $(H)=\operatorname{Inn}(H)$.

Proof of Proposition 4.2. By Lemmas 4.5 and 4.4. Out $(H)$ is residually finite. And $G / H$ embeds in $\operatorname{Out}(H)$ according to Lemma 4.3 , because $C_{G}(H) \subseteq E_{G}(H)=\{1\}$. Therefore, $G / H$ is residually finite as well.

Remark 4.6. We do not know whether one can remove the assumption that $H$ is nonelementary from Proposition 4.2 , because this is directly related to the well-known open question about the existence of non-(residually finite) hyperbolic groups.

Remark 4.7. The condition $E_{G}(H)=\{1\}$ in Proposition 4.2 is equivalent to the condition $E_{G}(G)=\{1\}$. In other words, it says that $G$ contains no non-trivial finite normal subgroups.

Indeed, since $E_{G}(H)$ is the (only) maximal finite subgroup of $G$ normalized by $H$ and $H \triangleleft G$, it is easy to see that $E_{G}(H) \triangleleft G$, hence $E_{G}(H) \leq E_{G}(G)$. Evidently, $E_{G}(G) \leq E_{G}(H)$, hence $E_{G}(H)=E_{G}(G)$.

We are now ready to prove Theorem 1.3 .

Proof of Theorem 1.3. The sufficiency is an immediate consequence of Proposition 4.1, because a normal subgroup is separable if and only if the quotient by it is residually finite.

In order to prove the necessity, suppose that $H$ is conjugacy separable. If $H$ is virtually cyclic, then $H$ is quasiconvex in $G$ (see [1, Cor. 3.4]). And since $H \triangleleft G$, by [1, Prop. 3.9], $H$ is either finite or has finite index in $G$. In either of these two cases, $G / H$ is residually finite: in the latter case this is obvious and in the former case this follows from the fact that $G$ is residually finite (since it is conjugacy separable), because any finite subset of a residually finite group is separable.

Thus we can assume that $H$ is non-elementary. Observe that $E_{G}(H)=\{1\}$ because $G$ is torsion-free. Consequently, $G / H$ is residually finite by Proposition 4.2 .

\section{Hereditarily CONJugacy SeParable Rips's CONStRuCtion}

Rips's construction, discovered by E. Rips [30], is a very useful tool, that turned out to be a rich source of counterexamples, allowing to find finitely generated subgroups of word hyperbolic groups with exotic properties (e.g., subgroups that are finitely generated but not finitely presented; finitely generated subgroups with undecidable membership problem; etc.). Briefly speaking, for every finitely presented group $P$, Rips's construction produces a hyperbolic group $G$ together with a finitely generated normal subgroup $N \triangleleft G$ 
such that $G / N \cong P$. In fact, in addition to being hyperbolic, the group $G$ can be made to enjoy other agreeable properties. For instance, in [32] Wise gave a modification of Rips's construction, in which the group $G$ is residually finite. More recently, Haglund and Wise [18] suggested a different version of Rips's construction, producing $G$ as the fundamental group of a compact non-positively curved square thin $\mathcal{V H}$-complex. In this case the group $G$ will be linear and will have separable quasiconvex subgroups (see [18]). Moreover, as we show below, $G$ will also be hereditarily conjugacy separable. In writing this paper we have become aware of the recent work of O. Cotton-Barratt and H. Wilton [11], where it is shown that the residually finite version of Rips's construction, originally suggested by Wise in [32], is also conjugacy separable.

Thin $\mathcal{V H}$-complexes were introduced by Wise in 33 . For our purposes, we only need to know three facts about them. The first fact is that if $G$ is a fundamental group of compact thin $\mathcal{V H}$-complex $\mathcal{X}$, then it is word hyperbolic. Indeed, as shown by Wise in [33], the universal cover $\widetilde{\mathcal{X}}$ is a $\operatorname{CAT}(0)$ space which contains no immersed flats. Hence $\pi_{1}(\mathcal{X})$ is word hyperbolic by a theorem of M. Bridson [5]. The second fact is that $\pi_{1}(\mathcal{X})$ is torsion-free (for instance, because it acts freely on the locally compact CAT(0) space $\widetilde{\mathcal{X}}$, and any finite group acting on such a space fixes at least one point - see [7, II.2.8]). The third fact, proved by Haglund and Wise in [18, tells us that $G=\pi_{1}(\mathcal{X})$ belongs to the class $\mathcal{A} \mathcal{V} \mathcal{R}$.

Theorem 5.1. Let $P$ be an arbitrary finitely presented group. Then there exist a torsionfree word hyperbolic group $G$ and a finitely generated (normal) subgroup $N \triangleleft G$ such that $G / N \cong P$. Moreover, such a group $G$ can be taken to satisfy all of the following conditions:

(i) $G$ is the fundamental group of a compact non-positively curved thin $\mathcal{V H}$-complex;

(ii) $G \in \mathcal{A} \mathcal{V} \mathcal{R}$;

(iii) $G$ is hereditarily conjugacy separable.

The above theorem is essentially due to Haglund and Wise (see [18]). Only the property (iii) is new, but it follows from (ii) and the fact that torsion-free hyperbolic groups from the class $\mathcal{A} \mathcal{V} \mathcal{R}$ are hereditarily conjugacy separable, which was proved by the second author in [25, Cor. 9.11].

Finally, we note that for a given finite presentation of $P$, a finite presentation for the group $G$ from Theorem 5.1 can be constructed explicitly (see [18, Thm. 10.1]).

\section{Constructing NON-Hereditarily CONJugacy SeParable Groups}

It is known that if a finitely presented group $Q$ has solvable word problem, then for any finite group $F$, any extension $P$ of $F$ by $Q$ also has solvable word problem (cf. [23, Lemma 4.7]). This group $P$ will also be finitely presented, as any (finitely presented)-by-(finitely presented) group - see [19, Lemma 1]. However, residual finiteness of $Q$ is not always passed to $P$. (The reader should recall and contrast with the fact that residual finiteness is passed to arbitrary subgroups and finite extensions.) 
More precisely, our algorithm for constructing (non-hereditarily) conjugacy separable groups takes as an input a short exact sequence of groups

$$
\{1\} \rightarrow F \rightarrow P \rightarrow Q \rightarrow\{1\}
$$

where $F$ is a finite group, $Q$ is residually finite and $P$ is finitely presented but not residually finite. The existence of such short exact sequences is non-trivial. We describe two ways to construct them below.

As observed by J. Corson and T. Ratkovich in [10], if one has a short exact sequence $\{1\} \rightarrow M \rightarrow R \rightarrow Q \rightarrow\{1\}$ of groups, where $M, Q$ are residually finite, $M$ is finitely generated and $R$ is not residually finite, then one can find a finite quotient $F$ of $M$ and an extension $P$, of $F$ by $Q$, which is not residually finite.

In the current literature we were able to find two examples of non-(residually finite) finitely presented (residually finite)-by-(residually finite) groups.

Example 6.1. In [12], P. Deligne proved that for every integer $n \geq 2$, there is a central extension $R$ of the infinite cyclic group $\langle a\rangle \cong \mathbb{Z}$ by the group of integral symplectic matrices $\operatorname{Sp}(2 n, \mathbb{Z})$, such that the element $a^{2}$ belongs to the kernel of every homomorphism from $R$ to a finite group (more precisely, $R=\widehat{\operatorname{Sp}(2 n, \mathbb{Z})}$ is the inverse image of $\operatorname{Sp}(2 n, \mathbb{Z})$ in the universal cover of $\operatorname{Sp}(2 n, \mathbb{R}))$. Consequently, for every $m \in \mathbb{N}$, the group $R_{m}:=$ $R /\left\langle a^{m}\right\rangle$ is a central extension of the cyclic group $\mathbb{Z} / m \mathbb{Z}$ by $\operatorname{Sp}(2 n, \mathbb{Z})$.

The group $\operatorname{Sp}(2 n, \mathbb{Z})$ is finitely presented (as an arithmetic subgroup of the algebraic group $\operatorname{Sp}(2 n, \mathbb{R})$ - see [29, Ch. 4.4, Thm. 4.2]) and residually finite (as any finitely generated subgroup of a linear group, according to Mal'cev's theorem [21]). Thus, the group $R_{m}$ is finitely presented and contains a central cyclic subgroup $C_{m}$ of order $m$, such that $R_{m} / C_{m} \cong \operatorname{Sp}(2 n, \mathbb{Z})$. And if $m \geq 3, R_{m}$ is not residually finite by the above theorem of Deligne.

The group $R_{2}$ may be residually finite, but in this case $R_{4}$, mapping onto $R_{2}$, contains a finite index subgroup $P$ that avoids the generator $c$ of the central subgroup $C_{4}$. By Deligne's result, $c^{2} \in P$, hence we have a short exact sequence $\{1\} \rightarrow\left\langle c^{2}\right\rangle_{2} \rightarrow P \rightarrow Q \rightarrow$ $\{1\}$, where $Q$ is a finite index subgroup of $\operatorname{Sp}(2 n, \mathbb{Z})$ (more precisely, $Q$ is the image of $P$ in $\operatorname{Sp}(2 n, \mathbb{Z}))$. Thus $Q$ is residually finite, $P$ is a finitely presented extension of $\mathbb{Z} / 2 \mathbb{Z}$ by $Q$, and $P$ is not residually finite (because $R_{4}$ is not).

Example 6.2. More recently, P. Hewitt [20] proved that there exists an extension $E$ of the free abelian group $A:=\mathbb{Z}^{3}$ by $\operatorname{SL}(3, \mathbb{Z})$ that is not residually finite (however, no explicit constructions for $E$ are known so far). It follows (see [10]), that for every sufficiently large $m \in \mathbb{N}$ there is a non-(residually finite) extension $E_{m}:=E / A^{m}$ of $A / A^{m} \cong(\mathbb{Z} / m \mathbb{Z})^{3}$ by $\mathrm{SL}(3, \mathbb{Z})$.

It is well known that $\mathrm{SL}(3, \mathbb{Z})$ is finitely presented and residually finite, therefore the short exact sequence $\{1\} \rightarrow(\mathbb{Z} / m \mathbb{Z})^{3} \rightarrow E_{m} \rightarrow \mathrm{SL}(3, \mathbb{Z}) \rightarrow\{1\}$ enjoys the required properties.

Let $B_{m}:=A / A^{m} \cong(\mathbb{Z} / m \mathbb{Z})^{3}$ denote the image of $A$ in $E_{m}$, and let $T_{m}:=C_{E_{m}}\left(B_{m}\right)$. Then $B_{m} \leq T_{m}$ and $\left|E_{m}: T_{m}\right|<\infty$ because $B_{m}$ is a finite normal subgroup of $E_{m}$. Thus 
$T_{m}$ is a central extension of $B_{m}$ by a finite index subgroup of $\mathrm{SL}(3, \mathbb{Z})$ (which is the image of $T_{m}$ under the epimorphism $E_{m} \rightarrow \mathrm{SL}(3, \mathbb{Z})$ ). As before, $T_{m}$ fails to be residually finite because it has finite index in the non-(residually finite) group $E_{m}$. We can now argue similarly to Example 6.1, to produce, for every $q \geq 2$, a central extension of the cyclic group $C_{q}$, of order $q$, by a finite index subgroup of $\mathrm{SL}(3, \mathbb{Z})$, which is not residually finite.

Main construction. Start with the short exact sequence (6.1) such that $F$ is a finite group, $Q$ is residually finite and $P$ is finitely presented but not residually finite. By Theorem 5.1 we can find a torsion-free hereditarily conjugacy separable hyperbolic group $G$ and a finitely generated normal subgroup $N \triangleleft G$ such that $G / N \cong P$. Thus there is an epimorphism $\psi: G \rightarrow P$ with $\operatorname{ker}(\psi)=N$. Let $H \leq G$ be the full preimage of $F$ under $\psi$. We have the following commutative diagram:

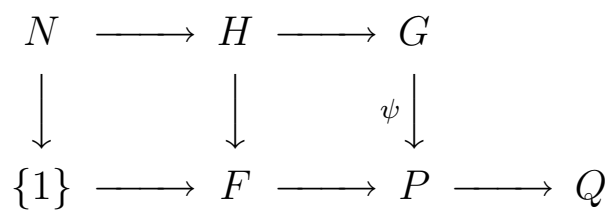

Then $H \triangleleft G, N \leq H$ and $H$ is finitely generated (since $H / N \cong F$ is finite). Observe that $G / H \cong P / F \cong Q$ is residually finite, hence $H$ is conjugacy separable according to Theorem 1.3. On the other hand, $G / N \cong P$ is not residually finite, and Theorem 1.3 implies that $N$ is not conjugacy separable. And since $N$ has finite index in $H$, we see that the group $H$ is conjugacy separable, but not hereditarily conjugacy separable.

Remark 6.3. The group $G$ in the above construction can be chosen from the class $\mathcal{V} \mathcal{R}$. Therefore the (non-hereditarily) conjugacy separable group $H$ will be a subgroup of some right angled Artin group $A$.

Indeed, by Theorem 5.1, the group $G$ from the main construction belongs to the class $\mathcal{A} \mathcal{V} \mathcal{R}$. Hence there is a finite index subgroup $G_{1} \leq G$ such that $G_{1} \in \mathcal{V} \mathcal{R}$. Note that $G_{1}$ is hereditarily conjugacy separable, torsion-free and hyperbolic, because all of these properties are inherited by finite index subgroups. Denote $N_{1}:=N \cap G_{1}, H_{1}:=H \cap G_{1}$, $P_{1}:=\psi\left(G_{1}\right), F_{1}:=P_{1} \cap F \triangleleft P_{1}$, and let $Q_{1}$ be the image of $P_{1}$ in $Q$. Clearly $\left|P: P_{1}\right|<\infty$, therefore $P_{1}$ cannot be residually finite. And since $G_{1} / N_{1} \cong P_{1}, G_{1} / H_{1} \cong Q_{1} \leq Q$, Theorem 1.3 yields that $H_{1}$ is conjugacy separable and $N_{1}$ is not conjugacy separable. Finally, we see that $\left|H_{1}: N_{1}\right|=\left|F_{1}\right| \leq|F|<\infty$.

Remark 6.4. The groups $N$ and $H$ from the main construction have solvable conjugacy problem.

This is an immediate consequence of Theorem 1.2 , because $Q \cong G / H$ and $P \cong G / N$ both have solvable word problem. Indeed, $Q$ is finitely presented and residually finite, hence the word problem in $Q$ is solvable by Mal'cev's result [22]. Therefore, $P$, being an extension of the finite group $F \cong H / N$ by $Q$, has solvable word problem as well. 


\section{Conjugacy separable fibre Products}

Let $G$ be a group. To every normal subgroup $N \triangleleft G$ one can associate the fibre product subgroup $T_{N} \leq G \times G$, defined by $T_{N}:=\left\{\left(g_{1}, g_{2}\right) \in G \times G \mid \psi\left(g_{1}\right)=\psi\left(g_{2}\right)\right\}$, where $\psi: G \rightarrow G / N$ is the natural epimorphism. It is not difficult to see that $T_{N}$ is the product of $N \times N \triangleleft G \times G$ with the diagonal subgroup $\left\{\left(g_{1}, g_{1}\right) \mid g_{1} \in G\right\} \leq G \times G$. The following remarkable "1-2-3 Theorem", discovered by G. Baumslag, M. Bridson, C. Miller and H. Short in [2], provides sufficient conditions for finite presentability of the fibre product:

Lemma 7.1. Suppose that $N$ is a normal subgroup of a group $G, Q:=G / N$ and $T_{N} \leq$ $G \times G$ is the fibre product associated to $N$. If $N$ is finitely generated, $G$ is finitely presented and $Q$ is of type $F_{3}$, then $T_{N}$ is finitely presented.

(Recall that a group is said to be of type $F_{n}$ if it has an Eilenberg-Maclane CW complex with only finitely many $k$-cells for each $k \leq n$.)

The above criterion together with the hereditarily conjugacy separable version of Rips's construction can be used to produce finitely presented (non-hereditarily) conjugacy separable groups. However, before doing this, we need to establish analogues of Propositions 4.1 and 4.2 for fibre products.

If $G$ is a group, $H \leq G$ and $x \in G$, the $H$-conjugacy class of $x$ in $G$ is, by definition, the set $x^{H}:=\left\{h^{-1} x h \mid h \in H\right\}$.

Lemma 7.2. Suppose $H$ is a finite index subgroup of a group $K$ and $x \in H$. If $x^{H}$ is closed in $\mathcal{P} \mathcal{T}(H)$ then $x^{K}$ is closed in $\mathcal{P} \mathcal{T}(K)$.

Proof. Choose $g_{1}, \ldots, g_{k} \in K$ so that $K=\bigsqcup_{i=1}^{k} H g_{i}$. Since $|K: H|<\infty$, any subset of $H$ which is closed in $\mathcal{P} \mathcal{T}(H)$, is also closed in $\mathcal{P} \mathcal{T}(K)$ (because any subgroup of finite index in $H$ also has finite index in $K)$. Therefore, $x^{H}$ is closed in $\mathcal{P} \mathcal{T}(K)$. Consequently, $x^{K}=\bigcup_{i=1}^{k} g_{i}^{-1} x^{H} g_{i}$ is closed in $\mathcal{P} \mathcal{T}(K)$ as a finite union of closed sets.

Lemma 7.3. Let $G_{1}$ and $G_{2}$ be hereditarily conjugacy separable groups. Then their direct product $G:=G_{1} \times G_{2}$ is also hereditarily conjugacy separable.

Proof. Consider any finite index subgroup $K \leq G$ and any $x=\left(x_{1}, x_{2}\right) \in K$. Then there exist finite index normal subgroups $N_{i} \triangleleft G_{i}, i=1,2$, such that $N:=N_{1} \times N_{2} \leq K$. Set $H:=\langle x\rangle N \leq K$, then $x \in H$, and $H$ has finite index in $K$ and in $G$.

Take any element $y=\left(y_{1}, y_{2}\right) \in H$ such that $y \notin x^{H}$. Then there is $j \in\{1,2\}$ such that $y_{j} \notin x_{j}^{N_{j}}$. Observe that $x_{j}^{N_{j}}=x_{j}^{H_{j}}$, where $H_{j}:=\left\langle x_{j}\right\rangle N_{j}$ is the image of $H$ under the canonical projection $\rho_{j}: G \rightarrow G_{j}$. Thus $x_{j}, y_{j} \in H_{j}$ and $y_{j} \notin x_{j}^{H_{j}}$. Now, since $\left|G_{j}: H_{j}\right| \leq\left|G_{j}: N_{j}\right|<\infty, H_{j}$ is conjugacy separable by the assumptions. Hence there is a finite group $Q$ and a homomorphism $\psi: H_{j} \rightarrow Q$ such that $\psi\left(y_{j}\right) \notin \psi\left(x_{j}\right)^{Q}$. Define the homomorphism $\varphi: H \rightarrow Q$ by $\varphi:=\psi \circ \rho_{j}$. Evidently, $\varphi(y)=\psi\left(y_{j}\right) \notin \psi\left(x_{j}\right)^{Q}=\varphi(x)^{Q}$.

Thus we have shown that $x^{H}$ is closed in $\mathcal{P} \mathcal{T}(H)$. Therefore, by Lemma $7.2, x^{K}$ is closed in $\mathcal{P} \mathcal{T}(K)$ for every $x \in K$. And we can conclude that $K$ is conjugacy separable, as required. 
The next statement is proved in [25, Cor. 11.2].

Lemma 7.4. Let $G$ be a hereditarily conjugacy separable group. Suppose that $H$ is a subgroup of $G$ such that the double coset $C_{G}(h) H$ is separable in $G$ for every $h \in H$. Then $H$ is conjugacy separable.

A group $G$ is said to be cyclic subgroup separable if for every $g \in G$ the cyclic subgroup $\langle g\rangle \leq G$ is closed in $\mathcal{P} \mathcal{T}(G)$.

Proposition 7.5. Let $H$ be a normal subgroup of a torsion-free hyperbolic group $G$. If $G$ is hereditarily conjugacy separable and $G / H$ is cyclic subgroup separable, then the corresponding fibre product $T_{H} \leq G \times G$ is conjugacy separable.

Proof. Denote $Q:=G / H$, let $\psi: G \rightarrow Q$ be the natural epimorphism, and let $\eta: G \times G \rightarrow$ $Q \times Q$ be the homomorphism defined by $\eta\left(g_{1}, g_{2}\right):=\left(\psi\left(g_{1}\right), \psi\left(g_{2}\right)\right)$ for all $\left(g_{1}, g_{2}\right) \in G \times G$. Consider any element $x=\left(x_{1}, x_{2}\right) \in T_{H}$. We will show that the double coset $C_{G \times G}(x) T_{H}$ is separable in $G \times G$, and then Lemma 7.4 will allow us to conclude that $T_{H}$ is conjugacy separable.

If $x_{1}=1$ (or $\left.x_{2}=1\right)$ in $G$, then $(G, 1) \leq C_{G}(x)\left((1, G) \leq C_{G}(x)\right)$, and since $T_{H}$ contains the diagonal subgroup of $G \times G$, we have $C_{G}(x) T_{H}=G \times G$. Thus, in this case the double coset $C_{G}(x) T_{H}$ is separable in $G \times G$.

So, we can suppose that both $x_{1}$ and $x_{2}$ are infinite order elements in $G$. Then $\mid C_{G}\left(x_{i}\right)$ : $\left\langle x_{i}\right\rangle \mid<\infty$ for $i=1,2$, and since $C_{G \times G}(x)=C_{G}\left(x_{1}\right) \times C_{G}\left(x_{2}\right)$ in $G \times G$, we see that $\left|C_{G \times G}(x):\left\langle x_{1}\right\rangle \times\left\langle x_{2}\right\rangle\right|<\infty$. Consequently, there exist elements $z_{1}, \ldots, z_{n} \in C_{G \times G}(x)$ such that $C_{G \times G}(x)=\bigsqcup_{i=1}^{n} z_{i}\left(\left\langle x_{1}\right\rangle \times\left\langle x_{2}\right\rangle\right)$.

Note that $\left\langle x_{1}\right\rangle \times\left\langle x_{2}\right\rangle=\left\langle\left(x_{1}, 1\right)\right\rangle\left\langle\left(x_{1}, x_{2}\right)\right\rangle$ in $G \times G$. Therefore, since $x=\left(x_{1}, x_{2}\right) \in T_{H}$, we have $\left(\left\langle x_{1}\right\rangle \times\left\langle x_{2}\right\rangle\right) T_{H}=\left\langle\left(x_{1}, 1\right)\right\rangle T_{H}$. It is easy to see that $\eta\left(T_{H}\right)=D$, where $D:=$ $\{(q, q) \mid q \in Q\}$ is the diagonal subgroup of $Q \times Q$, and $T_{H}=\eta^{-1}(D)$ is the full preimage of $D$ in $G \times G$. Hence $\left\langle\left(x_{1}, 1\right)\right\rangle T_{H}=\eta^{-1}(\langle a\rangle D)$, where $a:=\eta\left(\left(x_{1}, 1\right)\right)=\left(a_{1}, 1\right) \in Q \times Q$, $a_{1}:=\psi\left(x_{1}\right)$.

Observe that $\left(q_{1}, q_{2}\right) \in\langle a\rangle D$ in $Q \times Q$ if and only if $q_{1} q_{2}^{-1} \in\left\langle a_{1}\right\rangle$ in $Q$. Therefore, if $\left(q_{1}, q_{2}\right) \notin\langle a\rangle D$, we can use the assumption that $Q$ is cyclic subgroup separable to find a finite index normal subgroup $M \triangleleft Q$ with $q_{1} q_{2}^{-1} \notin\left\langle a_{1}\right\rangle M$. Hence $\left(q_{1}, q_{2}\right) \notin\langle a\rangle D(M \times M)$ in $Q \times Q$, and $M \times M$ has finite index in $Q \times Q$. Thus we have shown that the double coset $\langle a\rangle D$ is closed in $\mathcal{P} \mathcal{T}(Q \times Q)$. Since $\eta: G \times G \rightarrow Q \times Q$ is a continuous map (with respect to the corresponding profinite topologies), we can conclude that $\left\langle\left(x_{1}, 1\right)\right\rangle T_{H}=\eta^{-1}(\langle a\rangle D)$ is closed in $\mathcal{P} \mathcal{T}(G \times G)$.

In $G \times G$ we have $C_{G \times G}(x) T_{H}=\bigcup_{i=1}^{n} z_{i}\left(\left\langle x_{1}\right\rangle \times\left\langle x_{2}\right\rangle\right) T_{H}=\bigcup_{i=1}^{n} z_{i}\left(\left\langle\left(x_{1}, 1\right)\right\rangle T_{H}\right)$. Which implies that $C_{G \times G}(x) T_{H}$ is closed in $\mathcal{P} \mathcal{T}(G \times G)$ as a finite union of closed sets.

Finally, since $G \times G$ is hereditarily conjugacy separable by Lemma 7.3 , we can apply Lemma 7.4 to conclude that $T_{H}$ is conjugacy separable.

We now turn to an analogue of Proposition 4.2 . 
Proposition 7.6. Let $H$ be a finitely generated non-elementary normal subgroup of a hyperbolic group $G$, with $E_{G}(H)=\{1\}$. If the fibre product $T_{H} \leq G \times G$, associated to $H$, is conjugacy separable, then $G / H$ is residually finite.

Proof. Since $H$ is non-elementary and $E_{G}(H)=\{1\}$, there is an infinite order element $h_{1} \in H$ such that $E_{G}\left(h_{1}\right)=\left\langle h_{1}\right\rangle$ (see [27, Lemma 3.4]). In particular, $C_{G}\left(h_{1}\right)=\left\langle h_{1}\right\rangle \leq H$ in $G$.

Consider any element $g_{1} \in G \backslash H$, and set $g:=\left(g_{1}, 1\right) \in G \times G, h:=\left(h_{1}, h_{1}\right) \in G \times G$. Note that $g \notin T_{H}$ (as $\left.g_{1} \notin H\right)$, therefore the elements $h, f:=h^{g} \in H \times H \leq T_{H}$ are not conjugate in $T_{H}$ because $C_{G \times G}(h)=\left\langle h_{1}\right\rangle \times\left\langle h_{1}\right\rangle \leq T_{H}$. The group $T_{H}$ is conjugacy separable by the assumptions, hence there exist a finite group $F$ and a homomorphism $\zeta: T_{H} \rightarrow F$ such that $\zeta(f) \notin \zeta(h)^{F}$. Denote by $\varphi: H \times H \rightarrow F$ the restriction of $\zeta$ to $H \times H$; then $\varphi(f) \notin \varphi(h)^{F}$.

Since $H \times H$ is finitely generated, we can find a finite index subgroup $N$ of $H \times H$ such that $N \leq \operatorname{ker}(\varphi)$ and $N \triangleleft G \times G$. Define $M:=(H \times H) / N$, and let $\alpha: H \times H \rightarrow M$ be the natural epimorphism. Observe that, by definition, $\varphi$ factors through $\alpha$, hence

$$
\alpha(f) \notin \alpha(h)^{M} \text { in } M \text {. }
$$

Define the map $\xi: G \rightarrow A u t(M)$ by $\xi\left(x_{1}\right)(y):=\alpha\left(\left(x_{1} y_{1} x_{1}^{-1}, y_{2}\right)\right)$ for all $x_{1} \in G$ and all $y \in M$, where $\left(y_{1}, y_{2}\right) \in H \times H$ is any element satisfying $y=\alpha\left(\left(y_{1}, y_{2}\right)\right)$. Note that $\xi$ is a well-defined homomorphism because $\left(x_{1} y_{1} x_{1}^{-1}, y_{2}\right)=\left(x_{1}, 1\right)\left(y_{1}, y_{2}\right)\left(x_{1}, 1\right)^{-1}$ and $\left(x_{1}, 1\right) N\left(x_{1}, 1\right)^{-1}=N$. Evidently $\xi(H) \leq \operatorname{Inn}(M)$, therefore $\xi$ canonically gives rise to a homomorphism $\bar{\xi}: G / H \rightarrow \operatorname{Out}(M)$, where $\operatorname{Out}(M):=\operatorname{Aut}(M) / \operatorname{Inn}(M)$ is the group of outer automorphisms of $M$.

Finally, we have $\xi\left(g_{1}\right)(\alpha(h))=\alpha(f)$, which together with 7.1$)$ implies that $\xi\left(g_{1}\right) \notin$ $\operatorname{Inn}(M)$. Thus $\bar{\xi}\left(g_{1} H\right) \neq 1$ in the finite group $\operatorname{Out}(M)$. Since we started with an arbitrary element $g_{1} \in G \backslash H$, we can conclude that $G / H$ is residually finite.

Corollary 7.7. Suppose that $G$ is a torsion-free residually finite hyperbolic group, $H \triangleleft G$ is a finitely generated normal subgroup, and $T_{H} \leq G \times G$ is the associated fibre product. If $G / H$ is not residually finite, then $T_{H}$ is not conjugacy separable.

Proof. As we have already shown in the proof of Theorem 1.3 , if $H$ were elementary, then $G / H$ would be residually finite. Therefore $H$ is non-elementary. And since $G$ is torsionfree, it does not contain any non-trivial finite subgroups. Hence $E_{G}(H)=\{1\}$, and the claim follows from Proposition 7.6.

We finish this section with two remarks concerning the conjugacy problem in fibre products. Let $G$ be a finitely generated group. We will say that the membership problem to cyclic subgroups (MPCS) is uniformly decidable in $G$, if there is an algorithm, which takes on input any two elements $x, y \in G$ and determines whether or not $y \in\langle x\rangle$ in $G$. Clearly, the latter property is a natural "algorithmic" analogue of cyclic subgroup separability (indeed, for any finitely presented cyclic subgroup separable group $G$, MPCS will be uniformly decidable by Mal'cev's result [22]). 
We can now formulate the corresponding counterparts of Propositions 3.1 and 3.3 .

Proposition 7.8. Let $H$ be a normal subgroup of a torsion-free hyperbolic group $G$. If the quotient $G / H$ has uniformly decidable MPCS, then the corresponding fibre product $T_{H} \leq G \times G$ has solvable conjugacy problem.

Proof. We leave this as an exercise for the reader. It can be easily derived from the proofs of Proposition 3.1 and Proposition 7.5 .

The proof of the next statement can be extracted from [2, Thm. A', Lemma 3.3].

Proposition 7.9. Suppose that $G$ is a torsion-free hyperbolic group, $H \triangleleft G$ is a finitely generated normal subgroup, and $T_{H} \leq G \times G$ is the associated fibre product. If $T_{H}$ has solvable conjugacy problem then $G / H$ has solvable word problem.

Proof. Again this is an exercise in view of the proofs of Propositions 3.3 and 7.6 .

\section{Finitely PResented examples}

In this section we construct examples of finitely presented (non-hereditarily) conjugacy separable groups. However, before proceeding we need one more auxiliary statement.

Lemma 8.1. Suppose $N$ and $H$ are normal subgroups of a group $G$ such that $N \leq H$ and $|H: N|<\infty$. Let $T_{N}, T_{H} \leq G \times G$ be the corresponding fibre products. Then $T_{N} \leq T_{H}$ and $\left|T_{H}: T_{N}\right|=|H: N|$.

Proof. The inclusion of $T_{N}$ in $T_{H}$ is an immediate consequence of the definition of a fibre product. Set $P:=G / N$ and let $\eta: G \times G \rightarrow P \times P$ be the natural epimorphism with $\operatorname{ker}(\eta)=N \times N$. Then $\eta\left(T_{N}\right)$ is the diagonal subgroup $D$ of $P \times P$, and $\eta\left(T_{H}\right)=$ $\eta(H \times H) D=\eta((H, 1)) D$, where $(H, 1):=\left\{\left(h_{1}, 1\right) \mid h_{1} \in H\right\} \triangleleft G \times G$.

And since $\eta((H, 1)) \cap D=\{1\}$ in $P \times P, \eta\left(T_{H}\right)$ is a semidirect product of $\eta((H, 1))$ and $D$. Therefore $\left|\eta\left(T_{H}\right): \eta\left(T_{N}\right)\right|=\left|\eta\left(T_{H}\right): D\right|=|\eta((H, 1))|=|H: N|$. Recall that $\operatorname{ker}(\eta)=N \times N \leq T_{N} \leq T_{H}$, hence $T_{N}$ and $T_{H}$ are the full $\eta$-preimages of $\eta\left(T_{N}\right)$ and $\eta\left(T_{H}\right)$ in $G \times G$ respectively. Thus we can conclude that $\left|T_{H}: T_{N}\right|=\left|\eta\left(T_{H}\right): \eta\left(T_{N}\right)\right|=$ $|H: N|$.

To produce the example, establishing Theorem 1.1, we need to start with a short exact sequence of groups

$$
\{1\} \rightarrow F \rightarrow P \rightarrow Q \rightarrow\{1\}
$$

such that

(i) $|F|=m<\infty$;

(ii) $Q$ is of type $F_{3}$;

(iii) $Q$ is cyclic subgroup separable;

(iv) $P$ is not residually finite. 
Observe that the sequences given in Examples 6.1 and 6.2 possess all these properties. Indeed, the groups $Q$, arising there, are finite index subgroups of $\operatorname{Sp}(2 n, \mathbb{Z})$ or $\operatorname{SL}(3, \mathbb{Z})$. By a theorem A. Borel and J.-P. Serre [4] such $Q$ is of type $F_{n}$ for every $n \in \mathbb{N}$. On the other hand, $Q$ is cyclic subgroup separable because $Q \leq \mathrm{GL}(m, \mathbb{Z})$ for some $m \in \mathbb{N}$, and $\mathrm{GL}(m, \mathbb{Z})$ is cyclic subgroup separable (see [31, Thm. 5, p. 61]).

Now we can use Theorem 5.1 to find a hereditarily conjugacy separable torsion-free hyperbolic group $G$ and a finitely generated normal subgroup $N \triangleleft G$ such that $G / N \cong P$. Let $\psi: G \rightarrow P$ be the natural epimorphism with $\operatorname{ker}(\psi)=N$. Set $H:=\psi^{-1}(F) \triangleleft G$; then we have the same commutative diagram $(6.2)$ as before.

Let $T_{N}, T_{H} \leq G \times G$ be the fibre products associated to $N$ and $H$ respectively. Note that $|H: N|=|F|<\infty$, hence $H$ is also finitely generated. The group $G$ is finitely presented, as any hyperbolic group, and $G / H \cong Q$ is of type $F_{3}$. Therefore Lemma 7.1 allows to conclude that the group $T_{H}$ is finitely presented.

Finally, $T_{H}$ is conjugacy separable by Proposition 7.5, and $T_{N}$ is not conjugacy separable by Corollary 7.7. And, according to Lemma 8.1, $T_{N} \leq T_{H}$ and $\left|T_{H}: T_{N}\right|=|H: N|=$ $|F|=m$. Thus the group $T_{H}$ is a finitely presented (non-hereditarily) conjugacy separable group.

To achieve the first additional claim of Theorem 1.1, first assume that $m$ is a prime number. Apply the above algorithm to find the groups $F, P, Q, G, N$ and $H$ as before. According to Theorem 5.1, there is a finite index subgroup $G_{1} \leq G$ with $G_{1} \in \mathcal{V} \mathcal{R}$. Observe that $P_{1}:=\psi\left(G_{1}\right)$ has finite index in $P$, hence it cannot be residually finite, implying that $P_{1} \cap F \neq\{1\}$. But since $|F|=m$ is a prime, we can conclude that $F \leq P_{1}$. Consequently, after setting $N_{1}:=N \cap G_{1}$ and $H_{1}:=H \cap G_{1}$, we see that $N_{1}, H_{1} \triangleleft G_{1}$ and $\left|H_{1}: N_{1}\right|=|F|=m$.

Since the class of right angled Artin groups is closed under taking direct products, the group $G_{1} \times G_{1}$ will be a subgroup of some right angled Artin group. Let $T_{N_{1}}, T_{H_{1}} \leq$ $G_{1} \times G_{1}$ be the fibre products associated to $N_{1}$ and $H_{1}$ respectively. As we showed above, $T_{H_{1}}$ is finitely presented and conjugacy separable, $T_{N_{1}}$ is not conjugacy separable, and $\left|T_{H_{1}}: T_{N_{1}}\right|=m$.

Now, if $m$ is a composite number, write $m=m_{1} m_{2} \cdots m_{l}$, where $m_{j}$ is a prime for every $j=1, \ldots, l$. Apply the above construction to each $m_{j}$, finding a finitely presented conjugacy separable group $T_{j}$, which is a subgroup of some right angled Artin group $A_{j}$, and contains a non-(conjugacy separable) subgroup $S_{j}$ of index $m_{j}$. Define the direct products $T:=\prod_{j=1}^{l} T_{j}$ and $S:=\prod_{j=1}^{l} S_{j} \leq T$. Then $T$ is a finitely presented subgroup of the right angled Artin group $A:=\prod_{j=1}^{l} A_{j}$ and $|T: S|=m_{1} \cdots m_{l}=m$. It is easy to see that direct products of conjugacy separable groups are conjugacy separable, hence $T$ is conjugacy separable. On the other hand, $S$ is cannot be conjugacy separable, because $S_{1}$ is not conjugacy separable (and any retract of a conjugacy separable group is itself conjugacy separable - see [25, Lemma 9.3]).

We thus obtain 
Corollary 8.2. There exists finitely presented subgroups of right angled Artin groups that are conjugacy separable but not hereditarily conjugacy separable.

It is not difficult to show that if a group $Q$ has uniformly decidable MPCS, then for any finite group $F$, any extension $P$ of $F$ by $Q$ also has uniformly decidable MPCS. Therefore, Proposition 7.8 (together with Mal'cev's theorem mentioned right before it) yields the second additional claim of Theorem 1.1 .

Corollary 8.3. Both of the groups $T$ and $S$ above have solvable conjugacy problem.

\section{REFERENCES}

[1] J.M. Alonso, et al., Notes on word hyperbolic groups. Edited by H. Short. Group theory from a geometrical viewpoint (Trieste, 1990), 3-63, World Sci. Publ., River Edge, NJ, 1991.

[2] G. Baumslag, M.R. Bridson, C.F. Miller III, H. Short, Fibre products, non-positive curvature, and decision problems. Comment. Math. Helv. 75 (2000), no. 3, 457-477.

[3] O. Bogopolski, A. Martino, E. Ventura, Orbit decidability and the conjugacy problem for some extensions of groups. Preprint, 2007. arXiv:0712.3104

[4] A. Borel, J.-P. Serre, Cohomologie d'immeubles et de groupes S-arithmétiques. Topology 15 (1976), no. 3, 211-232.

[5] M.R. Bridson, On the existence of flat planes in spaces of nonpositive curvature. Proc. Amer. Math. Soc. 123 (1995), no. 1, 223-235.

[6] M.R. Bridson, On the subgroups of semihyperbolic groups. Essays on geometry and related topics, Vol. 1, 2, 85-111, Monogr. Enseign. Math., 38, Enseignement Math., Geneva, 2001.

[7] M.R. Bridson, A. Haefliger, Metric spaces of non-positive curvature. Grundlehren der Mathematischen Wissenschaften [Fundamental Principles of Mathematical Sciences], 319. Springer-Verlag, Berlin, 1999. xxii+643 pp.

[8] S.C. Chagas, P.A. Zalesskii, Finite index subgroups of conjugacy separable groups. Forum Mathematicum 21 (2009), no. 2, 347-353.

[9] D.J. Collins, C.F. Miller III, The conjugacy problem and subgroups of finite index. Proc. London Math. Soc. (3) (1977) vol. 34, no. 3, 535-556.

[10] J.M. Corson, T.J. Ratkovich, A strong form of residual finiteness for groups. J. Group Theory 9 (2006), no. 4, 497-505.

[11] O. Cotton-Barratt, H. Wilton, Conjugacy separability of 1-acylindrical graphs of free groups. Preprint, 2009. arXiv:0906.0101

[12] P. Deligne, Extensions centrales non résiduellement finies de groupes arithmétiques. C. R. Acad. Sci. Paris Sér. A-B 287 (1978), no. 4, A203-A208.

[13] É. Ghys and P. de la Harpe, eds., Sur les groupes hyperboliques d'après Mikhael Gromov. Progress in Mathematics, 83. Birkhäuser Boston, Inc., Boston, MA, 1990. xii+285 pp.

[14] A.V. Goryaga, Example of a finite extension of an FAC-group that is not an FAC-group (Russian). Sibirsk. Mat. Zh. 27 (1986), no. 3, 203-205.

[15] A.V. Gorjaga, A.S. Kirkinskiŭ, The decidability of the conjugacy problem cannot be transferred to finite extensions of groups (Russian). Algebra i Logika 14 (1975), no. 4, 393-406.

[16] M. Gromov, Hyperbolic groups. Essays in group theory, 75-263, Math. Sci. Res. Inst. Publ., 8, Springer, New York, 1987.

[17] E.K. Grossman, On the residual finiteness of certain mapping class groups. J. London Math. Soc. (2) $9(1974 / 75), 160-164$.

[18] F. Haglund, D.T. Wise, Special cube complexes. Geom. Funct. Anal. 17 (2008), no. 5, 1551-1620.

[19] P. Hall, Finiteness conditions for soluble groups. Proc. London Math. Soc. (3) 4 (1954), 419-436.

[20] P.R. Hewitt, Extensions of residually finite groups. J. Algebra 163 (1994), no. 3, 757-772. 
[21] A.I. Malcev, On faithful representations of infinite groups by matrices (Russian). Mat. Sb. 8 (1940) 405-422. English transl. in Amer. Math. Soc. Transl. Ser. 245 (1965) 1-18.

[22] A.I. Mal'cev, On homormorphisms onto finite groups (Russian). Uchen. Zap. Ivanovskogo Gos. Ped. Inst. 18 (1958), 49-60.

[23] C.F. Miller III, Decision problems for groups - survey and reflections. Algorithms and classification in combinatorial group theory (Berkeley, CA, 1989), 1-59, Math. Sci. Res. Inst. Publ., 23, Springer, New York, 1992.

[24] A. Minasyan, Groups with finitely many conjugacy classes and their automorphisms. Comm. Math. Helv. 84 (2009), No. 2, pp. 259-296.

[25] A. Minasyan, Hereditary conjugacy separability of right angled Artin groups and its applications. Preprint, 2009. arXiv:0905. 1282

[26] A. Minasyan, D. Osin, Normal automorphisms of relatively hyperbolic groups. Trans. of the AMS, to appear. arXiv:0809.2408

[27] A.Yu. Olshanskii, On residualing homomorphisms and G-subgroups of hyperbolic groups. Internat. J. Algebra Comput. 3 (1993), no. 4, 365-409.

[28] D.V. Osin, Relatively hyperbolic groups: intrinsic geometry, algebraic properties, and algorithmic problems. Mem. Amer. Math. Soc. 179 (2006), no. 843.

[29] V. Platonov, A. Rapinchuk, Algebraic groups and number theory. Translated from the 1991 Russian original by Rachel Rowen. Pure and Applied Mathematics, 139. Academic Press, Inc., Boston, MA, 1994. xii+614 pp.

[30] E. Rips, Subgroups of small cancellation groups. Bull. London Math. Soc. 14 (1982), no. 1, 45-47.

[31] D. Segal, Polycyclic groups. Cambridge Tracts in Mathematics, 82. Cambridge University Press, Cambridge, 1983. xiv+289 pp.

[32] D.T. Wise, A residually finite version of Rips's construction. Bull. London Math. Soc. 35 (2003), no. $1,23-29$.

[33] D.T. Wise, The residual finiteness of negatively curved polygons of finite groups. Invent. Math. 149 (2002), no. 3, 579-617.

School of Mathematics, University of Southampton, Highfield, Southampton, SO17 1BJ, UNITED KINGDOM.

E-mail address: A.Martino@soton.ac.uk

E-mail address: aminasyan@gmail.com 